\title{
Letter to Editor \\ Definition of Cycle and Episode in Rapid Cycling Bipolar Disorder: An Area of Debate in Diagnosis and Research
}

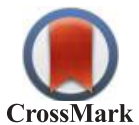

\section{*Elham Shirazi ${ }^{1}$, Amir Shabani ${ }^{2}$, Mitra Hakim Shooshtari ${ }^{1}$, Mohammad Ghadirivasfi ${ }^{3}$}

1. Child and Adolescent Psychiatrist, Associate Professor, Mental Health Research Center, School of Behavioral Sciences and Mental Health (Tehran Institute of Psychiatry), Iran University of Medical Sciences, Tehran, Iran.

2. Psychiatrist, Professor, Mental Health Research Center, Bipolar Disorders Research Group, Iran University of Medical Sciences, Tehran, Iran.

3. Psychiatrist, Associate Professor, Research Center for Addiction and Risky Behaviors (ReCARB), Iran University of Medical Sciences, Tehran, Iran.

\begin{tabular}{|c|c|}
\hline $\begin{array}{l}\text { Use your device to scan } \\
\text { and read the article online }\end{array}$ & tration: Shirazi E, Shabani A, Hakim Shooshtari M, Ghadiri Vasfi M. [Definition of Cycle and Episode in Rapid Cycling \\
\hline 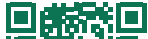 & Bipolar Disorder: An Area of Debate in Diagnosis and Research (Persian)]. Iranian Journal of Psychiatry and Clinical Psychology. \\
\hline 1304 & 2017; 23(3):254-259. https://doi.org/10.29252/NIRP.IJPCP.23.3.254 \\
\hline 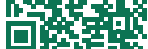 & dol]": https://doi.org/10.29252/NIRP.IJPCP.23.3.254 \\
\hline
\end{tabular}

Received: 02 Jul. 2017 Accepted: 20 Aug. 2017
Key words: Bipolar disorder, Cycle, Episode, Rapid cycling

\begin{abstract}
Kraepelin was the first who recognized the phenomenon of cycling in bipolar disorder in 1913. The term rapid cycling was proposed by Dunner and Fieve in 1974. Later on, Kramlinger and Post defined ultra-rapid cycling and ultradian cycling in 1996. Frequent rapid mood switches are an inherent characteristic of bipolar disorder, and rapid cycling is included as a course specifier for bipolar disorder in the fourth edition of the Diagnostic and Statistical Manual of Mental Disorders (DSM-IV). Although the terms cycle and episode are commonly used to delineate discrete periods of mood disorders, literature still suffers from the inconsistent and indistinct definition for them. Therefore, the reported prevalence of rapid cycling bipolar disorder shows a wide variability across studies, which ranges from $15 \%$ to $53.6 \%$ in adults, and $41.8 \%$ to $87.1 \%$ in children. Moreover, studies have used different definitions for rapid, ultra-rapid, and ultra-ultra-rapid/ultradian cycling, which has reduced the comparability of data across studies. However, the higher prevalence of rapid, ultra-rapid, and ultra-ultra-rapid/ultradian cycling reported in children with bipolar disorder, compared to adults, may make the pattern of cycling a key criterion in the diagnosis of early-onset bipolar disorder. Obviously, there is a significant need to stabilize a distinct specific definition for each of the terms episode, cycle, rapid cycling, ultra-rapid cycling, and ultra-ultra-rapid/ultradian cycling, based on the consensus of experts in bipolar disorder, in children and adults. In addition to increasing the value of research, using common terminologies and definitions enables comparison between studies and improves diagnostic criteria of rapid cycling bipolar disorder and its therapeutic and management plans in both the early- and adult-onset types.
\end{abstract}

* Corresponding Author:

Elham Shirazi, MD

Address: Department of Psychiatry, Mental Health Research Center, School of Behavioral Sciences and Mental Health (Tehran Institute of Psychiatry), Iran University of Medical Sciences, Tehran, Iran.

Tel: +98 (21) 66506853

E-mail: shirazi.e@iums.ac.ir 


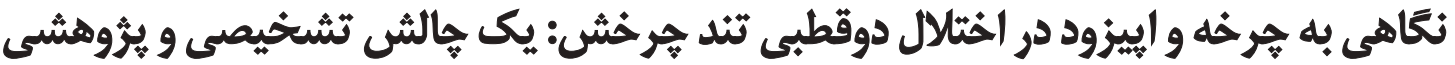

\author{
"الهام شيرازى'، امير شعبانى'، ميترا حكيم شوشترى'، محمد قديرى وصفى"
}

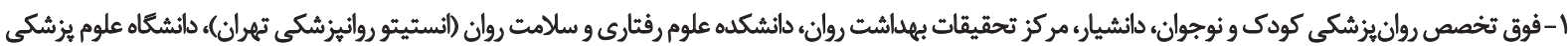

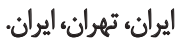

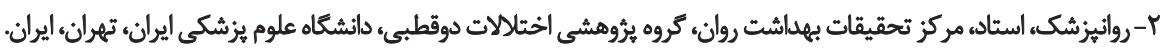

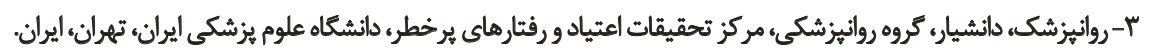

آنها استفاده شد. همجنين منابعى كه مقالهها به آن استناد داده

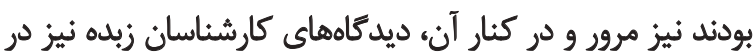

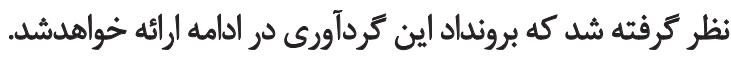

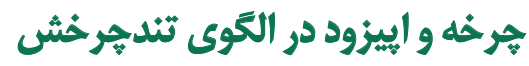

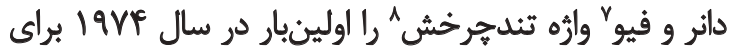

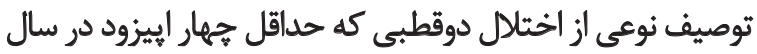

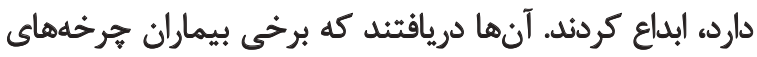

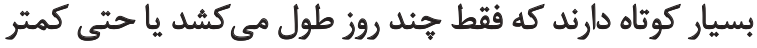

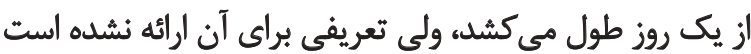

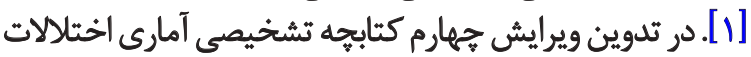

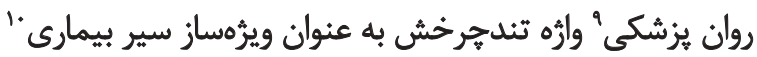

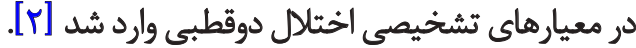

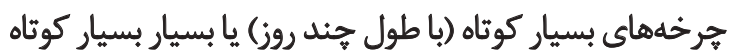

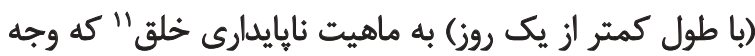

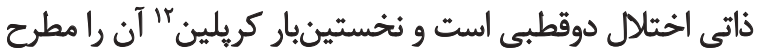

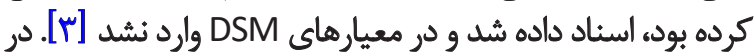

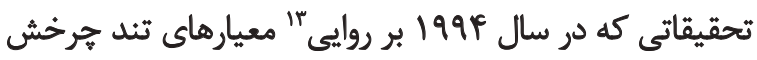

\section{Dunner \& Fieve}

8. Rapid Cycling

9. Diagnostic and Statistical Manual of Mental Disorders- $4^{\text {th }}$ Edition- DSM-IV

10. Course Specifier

11. Mood Lability

12. Kreapelin

13. Validity
در متون علمى نكاشتهشده درباره اختلال دوقطبى تند

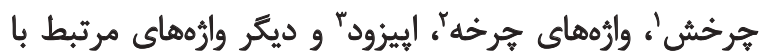

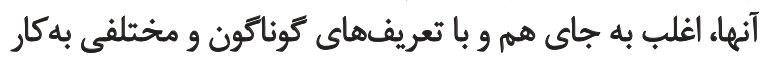

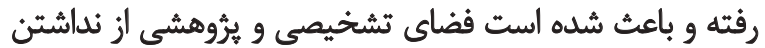

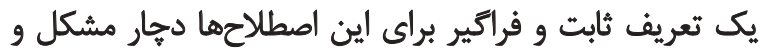

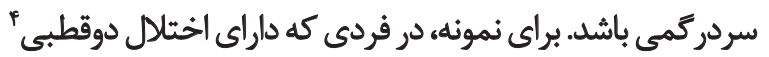

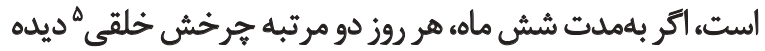

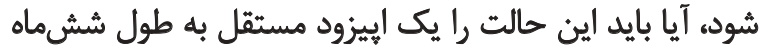

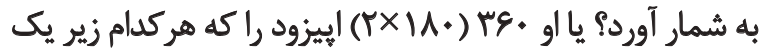

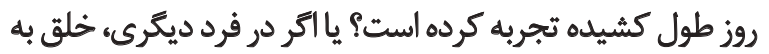

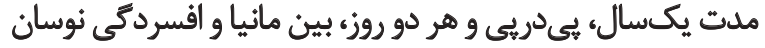

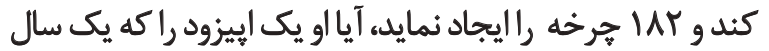

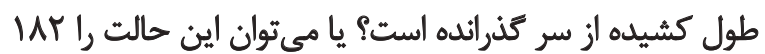

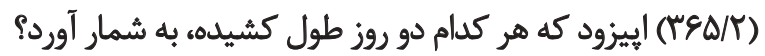

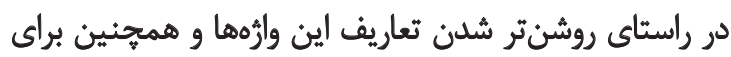

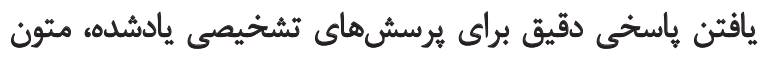

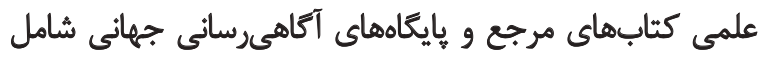

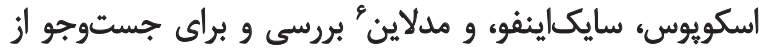
كليدوارههاي سائي cling, rapid cycling bipolar disorder, ultra rapid cycling, ultra ultra rapid cycling, ultradian cycling

1. Rapid cycling bipolar disorder

2. Cycle

3. Episode

4. Bipolar disorder

5. Mood cycling

6. Medline, PsychINFO, Scopus 
جدول ا. تعريف إيزود، جرخه، جرخشهاى فراتند و جرخشهاى فرافراتئلفراروز

\begin{tabular}{|c|c|c|c|c|}
\hline هرخش فرافرا تند/فراروز & هرخش فراتند & هرخه & اييزود & \\
\hline & 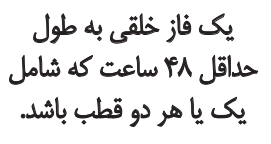 & 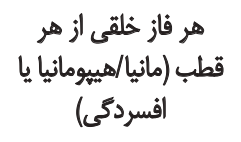 & & $\begin{array}{c}(14 \Delta)(10) \\
{[10]}\end{array}$ \\
\hline & & & 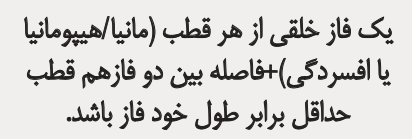 & $\begin{array}{l}\text { بائر و همكاران } \\
{[1 \% \text { (1999) }}\end{array}$ \\
\hline & & & 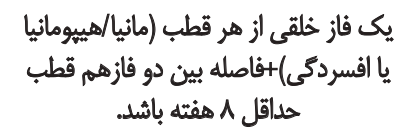 & 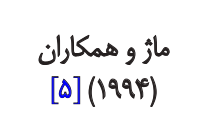 \\
\hline \multirow[t]{3}{*}{ 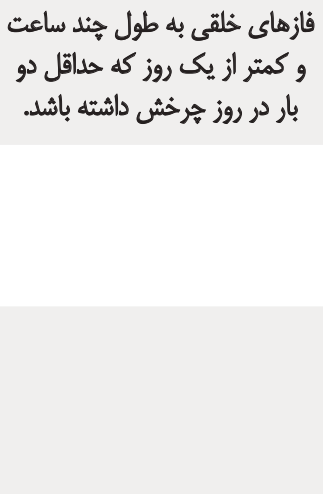 } & فازهاى خلقى به طول جند & & يكى فاز خلقى از هر قطب (مانيا/هييومانيا & 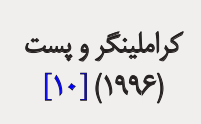 \\
\hline & & 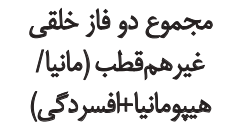 & يك فاز خلقى از هر قطب (مانيا/هييوماثيا & دائر (194) [ع] \\
\hline & & & 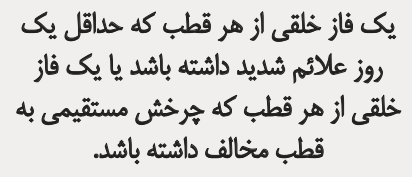 & $\begin{array}{l}\text { هاز و همكاران } \\
\text { [A] (1999) }\end{array}$ \\
\hline حداقل هعب جرخش در سال & Fef & & & $\begin{array}{l}\text { كلرو همكاران } \\
{[111](\%+\cdots)}\end{array}$ \\
\hline \multirow[t]{5}{*}{ حداقل يك فاز خلقي در روز براى } & حداقل f فاز خلقى در ماه & & & 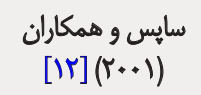 \\
\hline & & 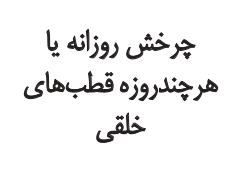 & 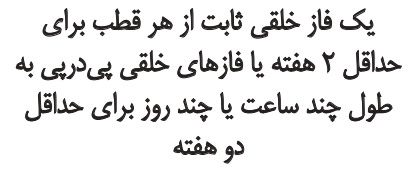 & 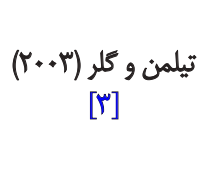 \\
\hline & 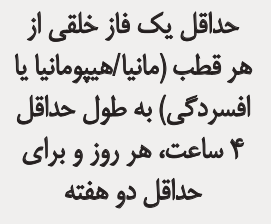 & & & 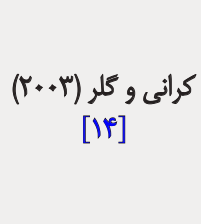 \\
\hline & & 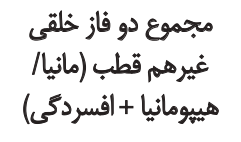 & يك فاز خلقى از هر قطب (ماثيا/هييومائيا & $\begin{array}{l}\text { بائر و همكاران } \\
{[V](r+1)}\end{array}$ \\
\hline & 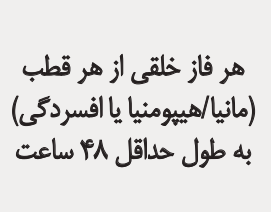 & 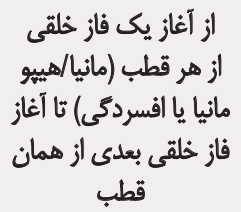 & & 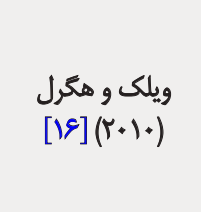 \\
\hline
\end{tabular}




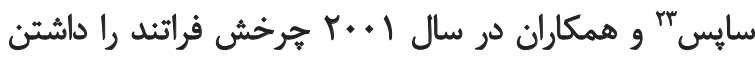

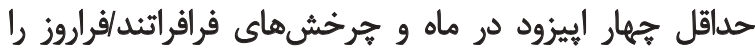

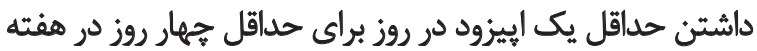
تعريف كردند [1Y]

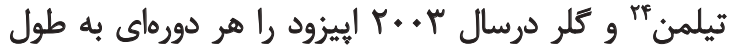

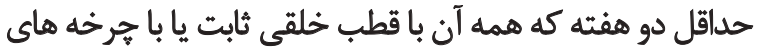

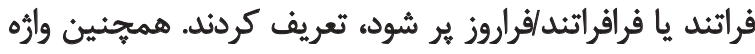

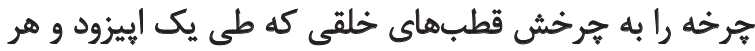

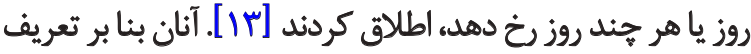

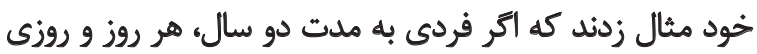

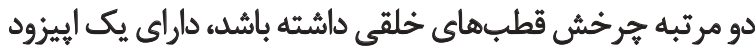

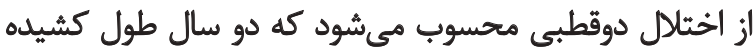

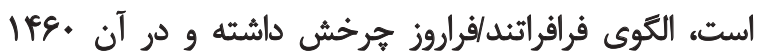

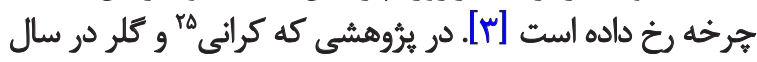

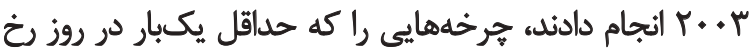

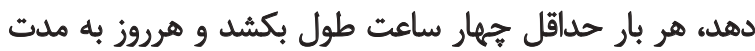

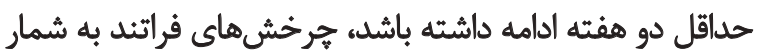

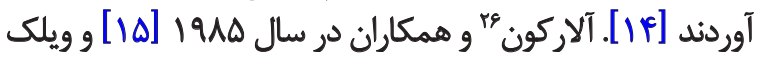

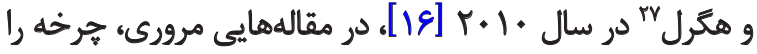

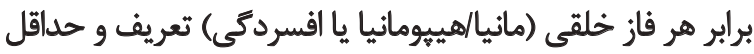

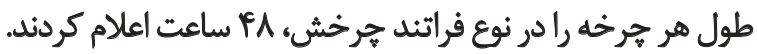
هرجند هنوز ارزش بالينى يا يُروهشى تغييراتى كه برُوهُشكران

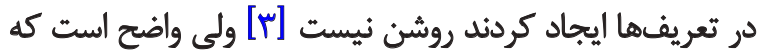

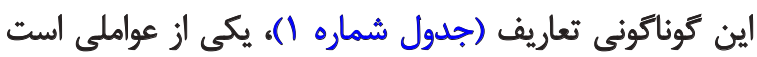

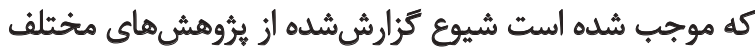

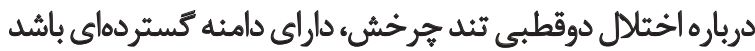

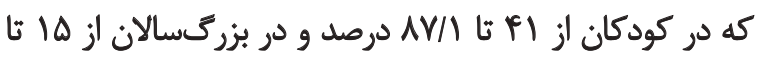

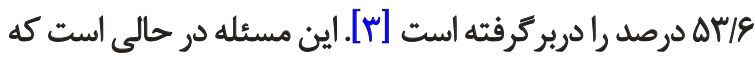

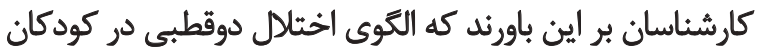

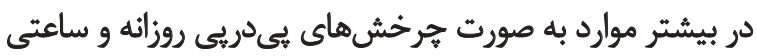

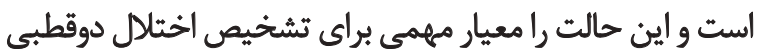

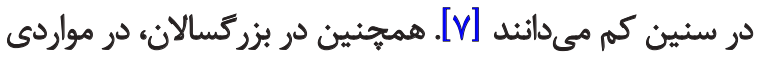

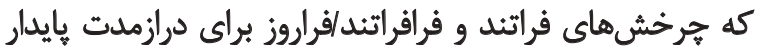

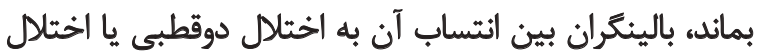

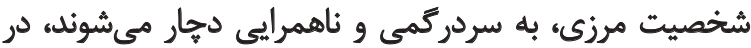

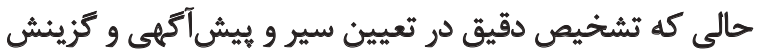

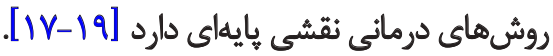

23. Suppes

24. Tillman

25. Craney

26. Alarcon

27. Wilk \& Hegerl

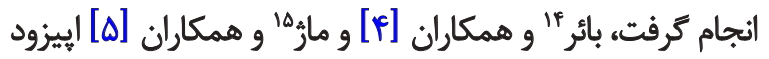

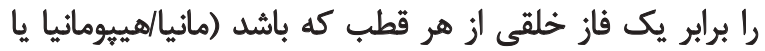

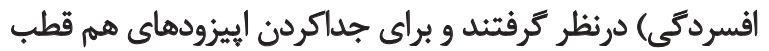

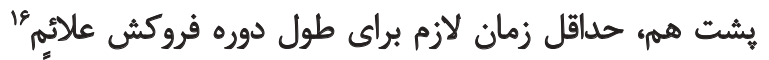

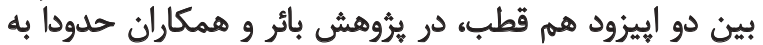

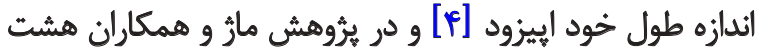

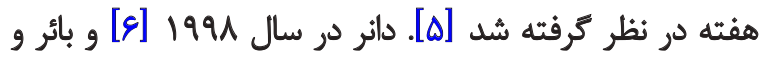

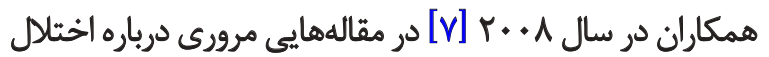

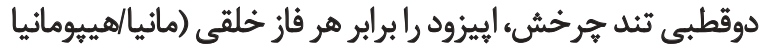

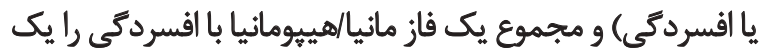
هرخه ناميدند.

بئا بر اين تعريف، آنها حداقل زمان لازم را براي يركردن يك يك

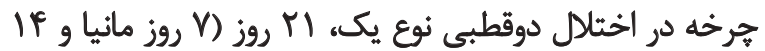

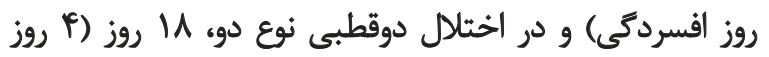

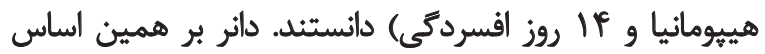

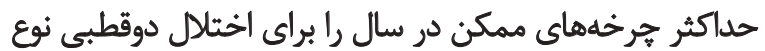

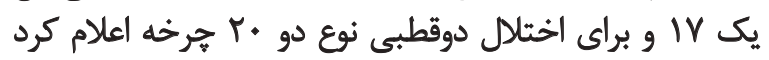

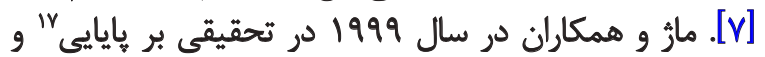

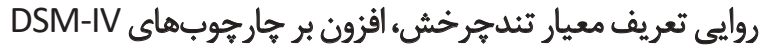

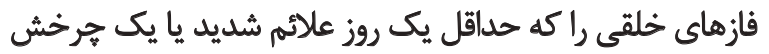

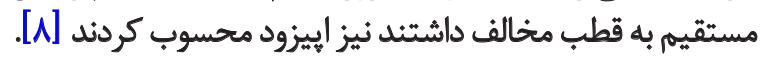
هرخه واييزود در الكّوى فراتندوفرافراتند/فراروز هرخش

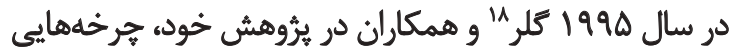

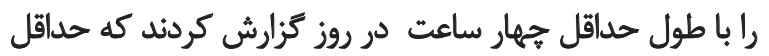

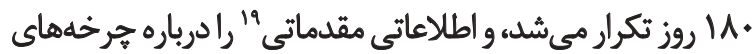

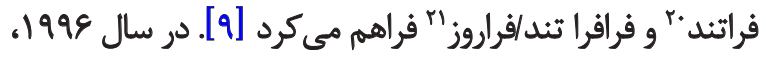

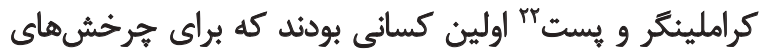

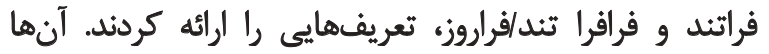

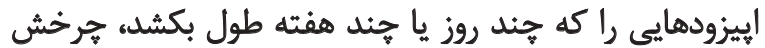

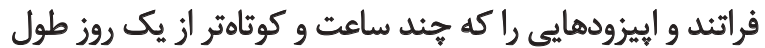

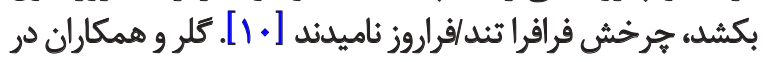

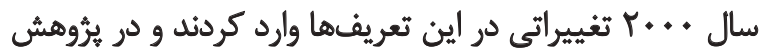

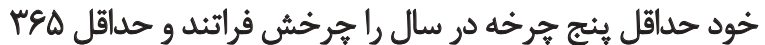

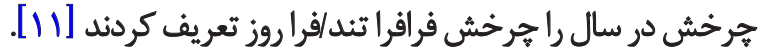

14. Bauer

15. Maj

16. Remission

17. Reliability

18. Geller

19. Pilot Data

20. Ultra Rapid

21. Ultra Ultra Rapid/Ulradian

22. Kramlinger \& Post 


\section{سخن بايانى}

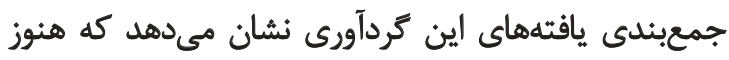

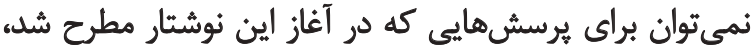

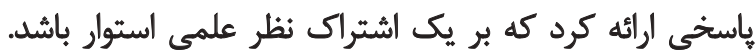

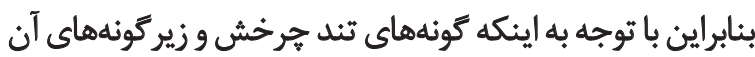

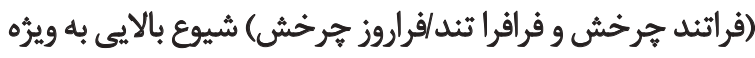

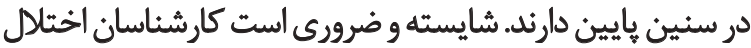

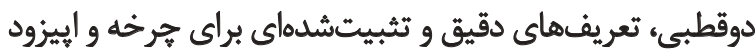

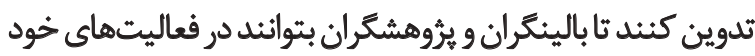
از تعاريف تشخيصى فراكير و يكسانى بيروى كنند.

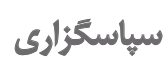

بنا به اظهار نويسنده مسئول مقاله، حمايت مالى از يُروهش و تعارض منافع وجود نداشته است. 


\section{References}

[1] Dunner DL, Fieve RR. Clinical factors in lithium carbonate prophylaxis failure. Archives of General Psychiatry. 1974; 30(2):229-33. doi: 10.1001/archpsyc.1974.01760080077013

[2] American Psychiatric Association. Diagnostic and Statistical Manual of Mental Disorders (DSM-IV). $4^{\text {th }}$ Edition. Washington, D.C.: American Psychiatric Association; 1994.

[3] Tillman R, Geller B. Definitions of rapid, ultrarapid, and ultradian cycling and of episode duration in pediatric and adult bipolar disorders: A proposal to distinguish episode from cycles. Journal of Child and Adolescent Psychiatry. 2003; 13(3):267-71. doi: $10.1089 / 104454603322572598$

[4] Bauer MS, Calabrese JR, Dunner DL, Post R, Whybrow PC, Gyulai L, et al. Multisite data reanalysis of the validity of rapid cycling as a course modifier for bipolar disorder in DSM-IV. American Journal of Psychiatry. 1994; 151(4):506-15. doi: 10.1176/ ajp.151.4.506

[5] Maj M, Magliano L, Pirozzi R, Marasco C, Guarneri M. Validity of rapid cycling as a course specifier for bipolar disorder. American Journal of Psychiatry. 1994; 151(7):1015-19. doi: 10.1176/ ajp.151.7.1015

[6] Dunner DL. Bipolar disorder in DSM-IV: Impact of inclusion of rapid cycling as a course modifier. Neuropsychopharmacology. 1998; 19(3):189-93. doi: 10.1016/S0893-133X(98)00024-4

[7] Bauer M, Beaulieu S, Dunner DL, Lafer B, Kupka R. Rapid cycling bipolar disorder- diagnostic concepts. Bipolar Disorders. 2008; 10(1p2):153-62. doi: 10.1111/j.1399-5618.2007.00560.x.

[8] Maj M, Prozzi R, Formicola AM, Tortorella A. Reliability and validity of four alternative definitions of rapid-cycling bipolar disorder. American Journal of Psychiatry. 1999; 159(9):1421-4. doi: 10.1176/ajp.156.9.1421

[9] Geller B, Sun K, Zimerman B, Luby J, Frazier J, Williams M. Complex and rapid-cycling in bipolar children and adolescents: A preliminary study. Journal of Affective Disorder. 1995; 34(4):25968. doi: 10.1016/0165-0327(95)00023-g

[10] Kramlinger KG, Post RM. Ultra-rapid and ultradian cycling in bipolar affective illness. British Journal of Psychiatry. 1996; 168(3):314-23. doi: 10.1192/bjp.168.3.314

[11] Geller B, Zimerman B, Williams M, Bolhofner K, Craney JL, DelBello MP, et al. Diagnostic characteristics of 93 cases of a prepubertal and early adolescent bipolar disorder phenotype by gender, puberty and comorbid attention deficit hyperactivity disorder. Journal of Child and Adolescent Psychopharmacology. 2000; 10(3):157-164. doi: 10.1089/10445460050167269

[12] Suppes T, Leverich GS, Keck PE, Nolen WA, Denicoff KD, Altshuler LL, et al. The Stanley Foundation of Bipolar Treatment Outcome Network II. Demographics and illness characteristics of the first 261 patients. Journal of Affective Disorder. 2001; 67(13):45-59. doi: 10.1016/s0165-0327(01)00432-3

[13] Shirazi E, Shabani A, Alaghband-Rad J. [Severe and nonepisodic irritabilities in children: Diagnostic debates and DSM role (Persian)]. Iranian Journal of Psychiatry and Clinical Psychology. 2014; 20(1):14-28.

[14] Craney JL, Geller B. A prepubertal and early adolescent bipolar disorder-I phenotype: Review of phenomenology and longitudinal course. Bipolar Disorders. 2003; 5(4):243-56. doi: 10.1034/j.1399-5618.2003.00044.x

[15] Alarcon RD. Rapid cycling affective disorders: A clinical review. Comprehensive Psychiatry. 1985; 26(6):522-40. doi: 10.1016/0010-440x(85)90019-7

[16] Wilk K, Hegerl U. Time of mood switches in ultra-rapid cycling disorder: A brief review. Psychiatry Research. 2010; 180(1):1-4. doi: 10.1016/j.psychres.2009.08.011

[17] Shabani A. [Strategies for decreasing false negative and positive diagnoses of bipolar disorders (Persian)]. Iranian Journal of Psychiatry and Clinical Psychology. 2009; 15(2):99-127.

[18] Shirazi E, Shahrivar Z. [Diagnostic debates of bipolar disorder: From the formal diagnostic criteria to the severe mood dysregulation (Persian)]. Iranian Journal of Psychiatry and Clinical Psychology. 2009; 15(2):128-46.

[19] Shabani A, Eftekhar M. [Borderline personality disorder is not a subtype of bipolar disorder (Persian)]. Iranian Journal of Psychiatry and Clinical Psychology. 2009; 15(2):147-58. 